\title{
Search for event bursts in XMASS-I associated with gravitational-wave events
}

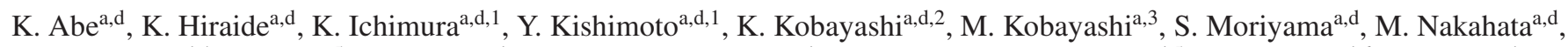

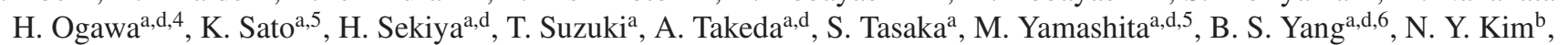

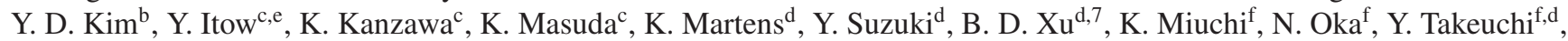
Y. H. Kim ${ }^{\mathrm{g}, \mathrm{b}}$, K. B. Lee ${ }^{\mathrm{g}}$, M. K. Lee ${ }^{\mathrm{g}}$, Y. Fukuda ${ }^{\text {h }}$, M. Miyasaka ${ }^{\mathrm{i}}$, K. Nishijima ${ }^{\mathrm{i}}$, K. Fushimi $^{\mathrm{j}}$, G. Kanzaki ${ }^{\mathrm{j}}$, S. Nakamura ${ }^{\mathrm{k}}$

\section{XMASS Collaboration*}

\author{
${ }^{a}$ Kamioka Observatory, Institute for Cosmic Ray Research, the University of Tokyo, Higashi-Mozumi, Kamioka, Hida, Gifu, 506-1205, Japan \\ ${ }^{b}$ Center for Underground Physics, Institute for Basic Science, 70 Yuseong-daero 1689-gil, Yuseong-gu, Daejeon, 305-811, South Korea \\ ${ }^{c}$ Institute for Space-Earth Environmental Research, Nagoya University, Nagoya, Aichi 464-8601, Japan \\ ${ }^{d}$ Kavli Institute for the Physics and Mathematics of the Universe (WPI), the University of Tokyo, Kashiwa, Chiba, 277-8582, Japan \\ ${ }^{e}$ Kobayashi-Maskawa Institute for the Origin of Particles and the Universe, Nagoya University, Furo-cho, Chikusa-ku, Nagoya, Aichi, 464-8602, Japan \\ ${ }^{f}$ Department of Physics, Kobe University, Kobe, Hyogo 657-8501, Japan \\ ${ }^{g}$ Korea Research Institute of Standards and Science, Daejeon 305-340, South Korea \\ ${ }^{h}$ Department of Physics, Miyagi University of Education, Sendai, Miyagi 980-0845, Japan \\ ${ }^{i}$ Department of Physics, Tokai University, Hiratsuka, Kanagawa 259-1292, Japan \\ ${ }^{j}$ Department of Physics, Tokushima University, 2-1 Minami Josanjimacho Tokushima city, Tokushima, 770-8506, Japan \\ ${ }^{k}$ Department of Physics, Faculty of Engineering, Yokohama National University, Yokohama, Kanagawa 240-8501, Japan
}

\section{Abstract}

We performed a search for event bursts in the XMASS-I detector associated with 11 gravitational-wave events detected during LIGO/Virgo's O1 and O2 periods. Simple and loose cuts were applied to the data collected in the full $832 \mathrm{~kg}$ xenon volume around the detection time of each gravitational-wave event. The data were divided into four energy regions ranging from keV to $\mathrm{MeV}$. Without assuming any particular burst models, we looked for event bursts in sliding windows with various time width from 0.02 to $10 \mathrm{~s}$. The search was conducted in a time window between -400 and $+10,000 \mathrm{~s}$ from each gravitational-wave event. For the binary neutron star merger GW170817, no significant event burst was observed in the XMASS-I detector and we set $90 \%$ confidence level upper limits on neutrino fluence for the sum of all the neutrino flavors via coherent elastic neutrino-nucleus scattering. The obtained upper limit was $(1.3-2.1) \times 10^{11} \mathrm{~cm}^{-2}$ under the assumption of a Fermi-Dirac spectrum with average neutrino energy of $20 \mathrm{MeV}$. The neutrino fluence limits for mono-energetic neutrinos in the energy range between 14 and $100 \mathrm{MeV}$ were also calculated. Among the other 10 gravitational wave events detected as the binary black hole mergers, a burst candidate with a $3.0 \sigma$ significance was found at 1801.95-1803.95 s in the analysis for GW151012. However, no significant deviation from the background in the reconstructed energy and position distributions was found. Considering the additional look-elsewhere effect of analyzing the $11 \mathrm{GW}$ events, the 'significance of finding such a burst candidate associated with any of them is $2.1 \sigma$.

Keywords: event burst, gravitational wave, neutrino, astroparticle, liquid xenon

\section{Introduction}

In 2015, the gravitational-wave (GW) signal from a binary black-hole merger was firstly detected by the Advanced

${ }^{*}$ E-mail address: xmass.publications14@km.icrr.u-tokyo.ac.jp .

${ }^{1}$ Now at Research Center for Neutrino Scientce, Tohoku Univeristy, Sendai 980-8578, Japan.

${ }^{2}$ Now at Waseda Research Institute for Science and Engineering, Waseda University, 3-4-1 Okubo, Shinjuku, Tokyo 169-8555, Japan.

${ }^{3}$ Now at Physics Department, Columbia University, New York, NY 10027, USA.

${ }^{4}$ Now at Department of Physics, College of Science and Technology, Nihon University, Kanda, Chiyoda-ku, Tokyo 101-8308, Japan.

${ }^{5}$ Now at Institute for Space-Earth Environmental Research, Nagoya University, Nagoya, Aichi 464-8601, Japan.

${ }^{6}$ Now at Center for Axion and Precision Physics Research, Institute for Basic Science, Daejeon 34051, South Korea.

${ }^{7}$ Department of Engineering Physics, Tsinghua University, Haidian District, Beijing 100084, China.
LIGO experiment [1]. During LIGO/Virgo's observing periods O1 (September 2015-January 2016) and O2 (November 2016-August 2017), 10 binary black-hole mergers and a binary neutron-star merger were observed [2]. Moreover, the electromagnetic counterparts were detected, for the first time, associated with the GW event from the binary neutron-star merger named GW170817 [3]; a short gamma-ray burst was detected $\sim 1.7 \mathrm{~s}$ after the GW event, and subsequent ultraviolet, optical, and infrared emissions were also observed [4]. Thus, a new era of the field of GW astronomy with multi-messenger observations has begun.

The follow-up searches for neutrino events associated with these GW events have also been conducted by gigantic neutrino detectors all over the world, however, no significant neutrino signal has been observed yet [5, 6, 7, , 8, 9, 10, 11, 12]. The neutrino follow-up searches are of interest because, for 
Table 1: List of the GW events during the whole XMASS-I data taking period. The data-taking situation of the XMASS-I detector in a time window between -400 and $+10,000 \mathrm{~s}$ from each $\mathrm{GW}$ event $\left(t_{\mathrm{GW}}\right)$ is also noted.

\begin{tabular}{lcc}
\hline \hline GW event & GW detection time $t_{\mathrm{GW}}$ (UTC) & Data-taking situation of XMASS-I \\
\hline GW150914 & Sep. 14, 2015 09:50:45 & Continuous data-taking \\
GW151012 & Oct. 12, 2015 09:54:43 & No data in $1,183<t-t_{\mathrm{GW}}<1,583 \mathrm{~s}$ due to run change \\
GW151226 & Dec. 26, 2015 03:38:53 & No data in $4,191<t-t_{\mathrm{GW}}<4,388$ s due to run change \\
GW170104 & Jan. 04, 2017 10:11:58 & No data in 196 $<t-t_{\mathrm{GW}}<275$ s due to run change \\
GW170608 & Jun. 08, 2017 02:01:16 & No data in $t-t_{\mathrm{GW}}>6,339$ s due to detector calibration \\
GW170729 & Jul. 29, 2017 18:56:29 & Continuous data-taking \\
GW170809 & Aug. 09, 2017 08:28:21 & Continuous data-taking \\
GW170814 & Aug. 14, 2017 10:30:43 & Continuous data-taking \\
GW170817 & Aug. 17, 2017 12:41:04 & Continuous data-taking \\
GW170818 & Aug. 18, 2017 02:25:09 & Continuous data-taking \\
GW170823 & Aug. 23, 2017 13:13:58 & Continuous data-taking \\
\hline \hline
\end{tabular}

instance, there are some theoretical predictions of emission of neutrinos with energy of a few tens $\mathrm{MeV}$ [13, 14], and much higher-energy neutrinos [15, 16] are expected from binary neutron-star mergers. The expected range for detecting several neutrinos from a binary neutron star merger is, however, $<10 \mathrm{Mpc}$ with a future megaton-scale water Cherenkov detector [13]. There also exist scenarios of production of short gamma-ray bursts [17] or ultrahigh-energy neutrinos [18] from binary black-hole mergers.

XMASS-I is a large single-phase liquid xenon (LXe) detector located underground (2700 m water equivalent) at the Kamioka Observatory in Japan [19]. It is a multi-purpose detector suitable for detecting particles with energy deposition in the wide energy range from $\mathrm{keV}$ to $\mathrm{MeV}$ under an ultra-low background environment. The XMASS-I detector accumulated data with a stable condition continuing from November 2013 until February 2019 , resulting in the entire data set with a total live time of 4.4 years. Using the XMASS-I data, various searches for astroparticles such as dark matter particles [20, 21, 22, 23], solar axions [24], and solar Kaluza-Klein axions [25] have been performed. Furthermore, the possibility to detect galactic supernova neutrinos via coherent elastic neutrino-nucleus scattering (CEvNS) has been studied [26].

In this paper, we report results from a search for event bursts in the XMASS-I detector associated with the $11 \mathrm{GW}$ events detected during LIGO/Virgo's $\mathrm{O} 1$ and $\mathrm{O} 2$ periods.

\section{XMASS-I detector}

The XMASS-I detector holds an active target of $832 \mathrm{~kg}$ of LXe inside a pentakis-dodecahedral copper structure that hosts 642 inward-looking 2-inch Hamamatsu R10789 photomultiplier tubes (PMTs) on its approximately spherical inner surface at a radius of about $40 \mathrm{~cm}$. The photocathode coverage of the inner surface is $62.4 \%$. The LXe detector is placed at the center of a cylindrical water Cherenkov detector. The outer detector, which is $11 \mathrm{~m}$ in height and $10 \mathrm{~m}$ in diameter, is equipped with 72 20-inch Hamamatsu H3600 PMTs. This detector acts as an active veto counter for cosmic-ray muons as well as a passive shield against neutrons and $\gamma$-rays from the surrounding rocks.

Data acquisition is triggered if at least four inner-detector PMTs record a signal within $200 \mathrm{~ns}$ or if at least eight outerdetector PMTs register a signal within $200 \mathrm{~ns}$. A $50 \mathrm{MHz}$ clock is used to measure the time difference between triggers. One-pulse-per-second (1PPS) signals from the global positioning system (GPS) are fed as triggers for precise time stamping. The GPS 1PPS triggers are also used to flash the LED for the PMT gain monitoring.

The gains of the PMTs are monitored weekly using a blue LED embedded in the inner surface of the detector. The scintillation yield response is traced with a ${ }^{57} \mathrm{Co}$ source [27] inserted along the central vertical axis of the detector every week or two. Through measurements with the ${ }^{57} \mathrm{Co}$ source at the center of the detector volume, the photoelectron (PE) yield was determined to be $\sim 15 \mathrm{PE} / \mathrm{keV}$ for $122 \mathrm{keV} \gamma$-rays. The nonlinear response of the scintillation yield for electron-mediated events in the detector was calibrated over the energy range from $5.9 \mathrm{keV}$ to $2614 \mathrm{keV}$ with ${ }^{55} \mathrm{Fe},{ }^{241} \mathrm{Am},{ }^{109} \mathrm{Cd},{ }^{57} \mathrm{Co},{ }^{137} \mathrm{Cs},{ }^{60} \mathrm{Co}$, and ${ }^{232} \mathrm{Th}$ sources. In this paper, the energy above $2614 \mathrm{keV}$ is extrapolated using the energy scale derived by the ${ }^{232}$ Th calibration. Hereinafter, this calibrated energy is represented as $\mathrm{keV}_{\mathrm{ee}}$ where the subscript stands for the electron-equivalent energy.

The timing offsets for the PMT channels owing to the differences in their cable lengths and the electronic responses were also traced by the ${ }^{57}$ Co calibration.

\section{Data set and event selection}

Table 1 shows a list of the GW events detected during LIGO/Virgo's O1 and $\mathrm{O} 2$ periods and the data-taking situation of the XMASS-I detector around the detection time of each GW event. The event burst search is conducted in a time window between -400 and $+10,000$ s from each GW event. This search window is motivated by two reasons. The time window within $\pm 400 \mathrm{~s}$ from each GW event is considered in the search for neutrinos as described in Sec. 5. In addition, the extended time 


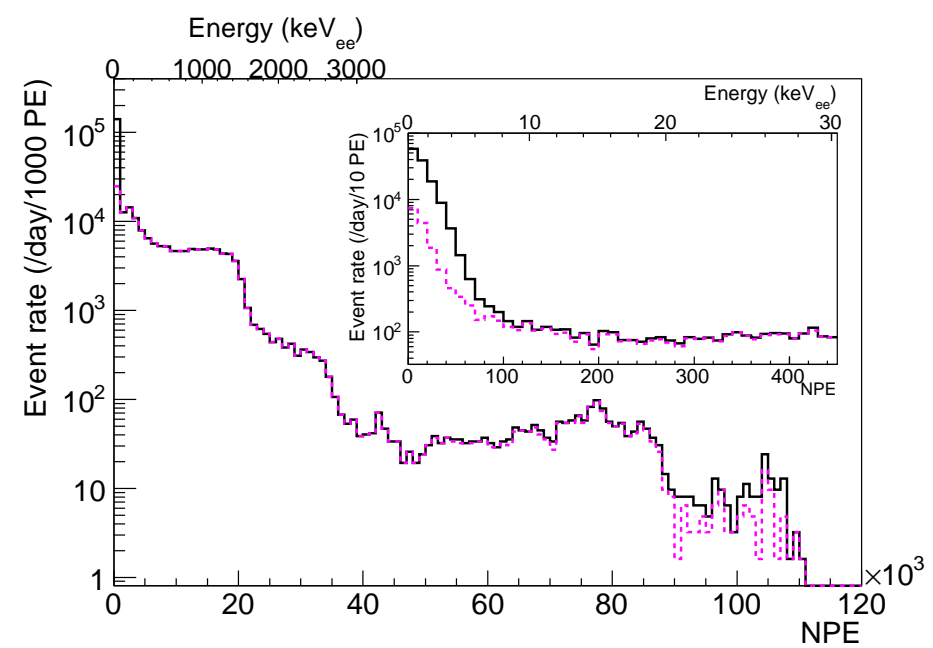

Figure 1: Observed number of PE spectra in the pre-search time window for the GW170817 event before (black solid) and after (magenta dashed) the event selection. The corresponding $\mathrm{keV}_{\mathrm{ee}}$ energy scale is shown at the top of the figure.

window up to $10,000 \mathrm{~s}$ is considered in the model independent search because more massive particles, axion-like particles for instance, might arrive later timing. For the GW151012, GW151226, and GW170104 events, there exists a-few-minute dead time due to run change. For the GW170608, data-taking stopped 6,339 s after the GW event for the detector calibration. Otherwise, data were taken continuously during the search window.

We use the full $832 \mathrm{~kg}$ of xenon as an active target in this analysis. In order to perform a search for event bursts with a minimal bias, only simple and loose cuts are applied. Events with four or more hits in the inner-detector without an associated outer-detector trigger are initially selected. We have then applied four selection cuts that mostly remove obvious backgrounds. To remove events caused by after-pulses in the PMTs following bright events, one requires that the standard deviation of the inner-detector hit timing distribution is less than $100 \mathrm{~ns}$, and that the time elapsed since the previous inner-detector event $\left(d T_{\text {pre }}\right)$ is at least $200 \mu$ s. To remove events due to Cherenkov light emission by the $\beta$-rays from ${ }^{40} \mathrm{~K}$ in the PMT photocathode, events in which more than $80 \%$ of the PMT hits arrive in the first $20 \mathrm{~ns}$ are discarded. For this analysis, the $d T_{\text {pre }}$ and Cherenkov cuts are loosen compared to other analyses in XMASS-I. The detection efficiency after those cuts will be described later.

Figure 1 shows the observed number of PE (NPE) spectra in the pre-search time window for the GW170817 event before and after the event selection. The pre-search window is defined as a time window between $-20,000$ and $-400 \mathrm{~s}$ from each GW event to estimate the background rate. The cuts mainly remove events below $100 \mathrm{PE}$ as well as a fraction of $\alpha$-ray events from the ${ }^{222} \mathrm{Rn}$ daughter, ${ }^{214} \mathrm{Po}$. Then, the data are divided into four NPE ranges: $<450$ (referred to as Low-E), 450-4500 (Middle-E), 4500-45000 (High-E), and >45000 (Very HighE, or V. H. E. hereinafter) corresponding to energy ranges of

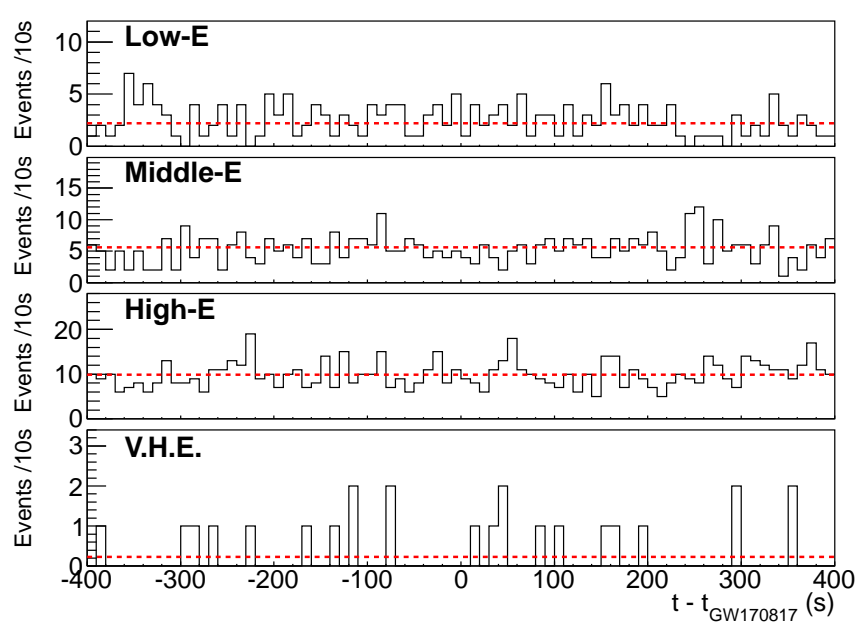

Figure 2: Event rate history within \pm 400 s from GW170817 (August 17, 2017, 12:41:04 UTC). From top to bottom, the Low-E, Middle-E, High-E, and V. H. E. samples are shown. The horizontal dashed lines correspond to the average background event rate estimated from the pre-search window.

approximately $<30,30-300,300-3500$, and $>3500 \mathrm{keV}_{\mathrm{ee}}$, respectively. The average event rate in these four energy ranges are estimated from the pre-search window, to be $0.223 \pm 0.004$, $0.559 \pm 0.006,0.987 \pm 0.008$, and $0.023 \pm 0.001 \mathrm{~Hz}$, respectively.

\section{Model independent event burst search}

\subsection{Results for the GW170817 binary neutron star merger}

Figures 2 shows the event rate history within $\pm 400 \mathrm{~s}$ from GW170817 (August 17, 2017 12:41:04 UTC). The event rate history in a wider time range up to $+10,000$ s from the $\mathrm{GW}$ event is shown in Fig. 3. The average background event rates estimated from the pre-search window are shown as horizontal dashed lines. A close-up event rate history within $\pm 10 \mathrm{~s}$ from GW170817 is also shown in Fig. 4.

To search for event bursts without assuming any particular burst model, the number of events in a sliding time window is scanned for each energy range. The coincidence time window is slid at a step of $0.01 \mathrm{~s}$ and various width of the window $\left(t_{\mathrm{width}}\right)$ are tested: $0.02,0.04,0.1,0.2,0.4,1,2,4$, and $10 \mathrm{~s}$. The range of the width of the sliding window is determined referring to the neutrino emission model discussed in ref. [13]; the neutrino emission peak continues $10-100 \mathrm{~ms}$ with a long cooling time of $2-3 \mathrm{~s}$ is predicted. For each energy region, a test statistics (TS) is constructed under the null hypothesis as

$$
\mathrm{TS}=2 \ln \left[\left(\frac{T_{\text {search }}}{t_{\text {width }}}\right) \times P\left(\mu_{\mathrm{bg}} \mid N_{\mathrm{obs}}\right)\right],
$$

where $P(\mu \mid N)=\mu^{N} e^{-\mu} / N$ ! is the Poisson probability, and $N_{\text {obs }}$ and $\mu_{\mathrm{bg}}$ are the observed number of events and the expected number of background events in a time window. The factor $T_{\text {search }} / t_{\text {width }}$ corrects for the look-elsewhere effect due to choosing a time window of width $t_{\text {width }}$ from the whole search window $T_{\text {search}}$. 


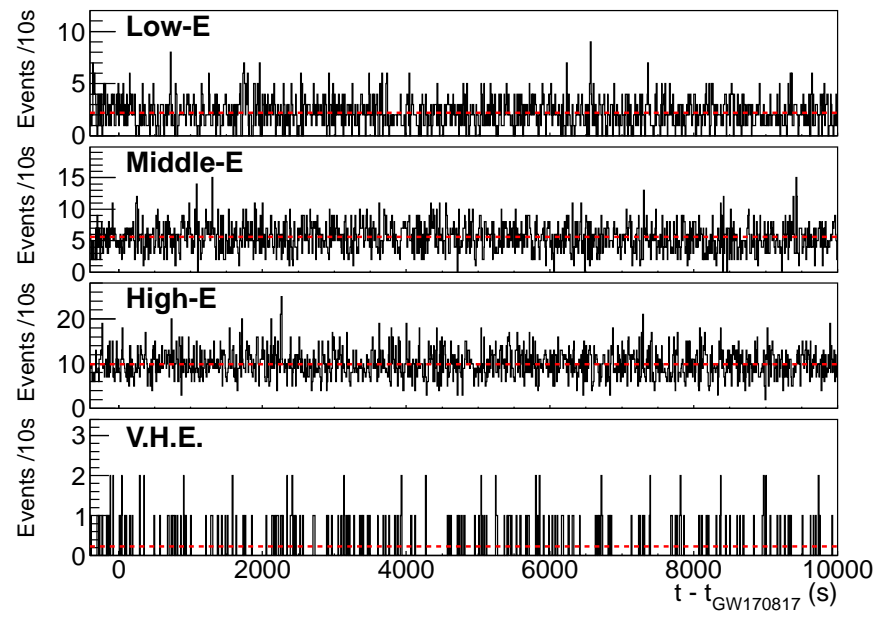

Figure 3: Event rate history in a wider time range up to $+10,000 \mathrm{~s}$ from GW170817 (August 17, 2017 12:41:04 UTC). From top to bottom, the Low-E, Middle-E, High-E, and V. H. E. samples are shown. The horizontal dashed lines correspond to the average background event rate estimated from the pre-search window.

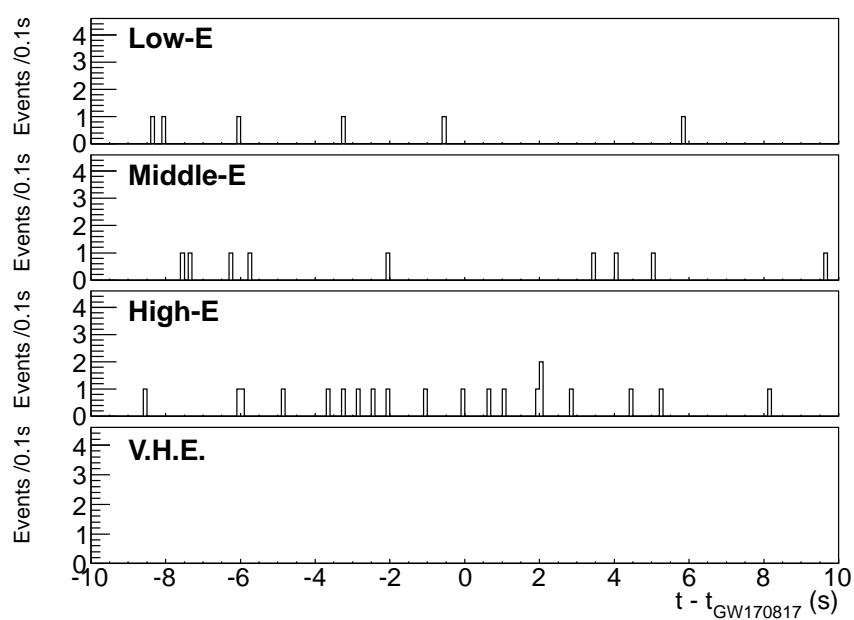

Figure 4: Event rate history within $\pm 10 \mathrm{~s}$ from GW170817 (August 17, 2017 12:41:04 UTC). From top to bottom, the Low-E, Middle-E, High-E, and V. H. E. samples are shown.
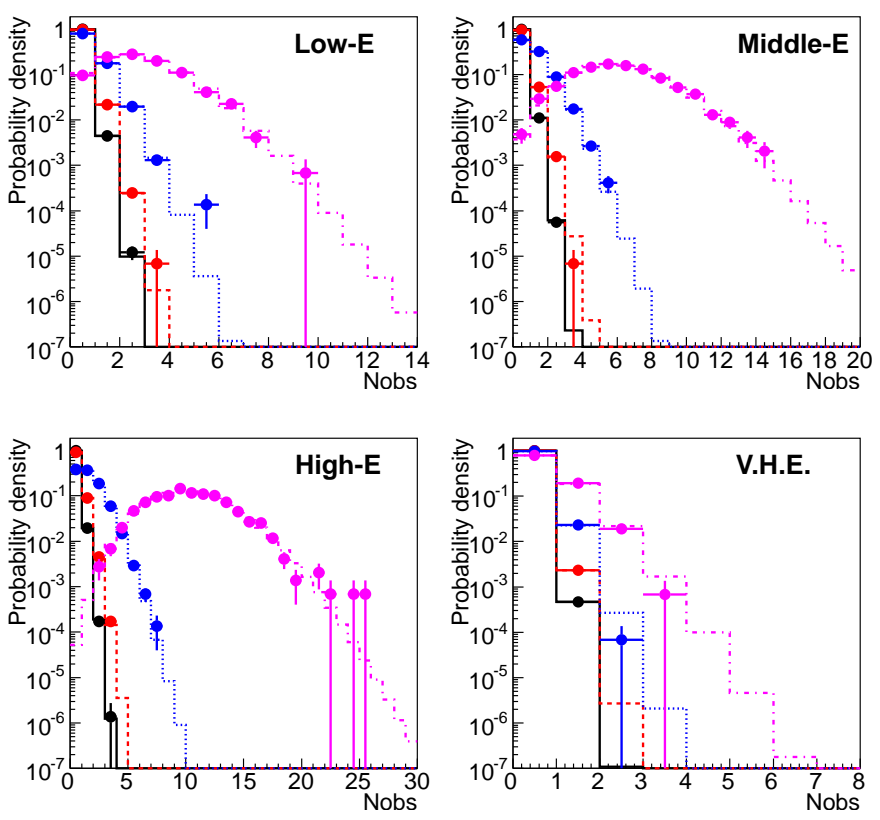

Figure 5: Distributions of the observed number of events $\left(N_{\mathrm{obs}}\right)$ in time windows of width $0.02 \mathrm{~s}$ (black solid), $0.1 \mathrm{~s}$ (red dashed), $1 \mathrm{~s}$ (blue dotted) and $10 \mathrm{~s}$ (magenta dash-dotted) in each energy range. The points are the data from the pre-search window and the histograms are obtained from the simulation described in the text.

At each timing $t-t_{\mathrm{GW}}$, we first minimize the test statistics as a function of $t_{\text {width. }}$. Then, we scan the test statistics as a function of the timing $t-t_{\mathrm{GW}}$ to find minimums as possible burst candidates.

To calculate a global significance of each candidate, we estimate the probability distribution of the test statistics under the null hypothesis by performing the same analysis on 100,000 dummy data sets. In each dummy data set, events are randomly generated based on the average event rate estimated in the pre-search window for each energy region. Possible timecorrelated backgrounds due to short-time consecutive decays of radioisotopes in the detector material are also considered. ${ }^{222} \mathrm{Rn}$ in the LXe decays through the ${ }^{222} \mathrm{Rn}\left(T_{1 / 2}=3.82 \mathrm{~d}\right)-{ }^{218} \mathrm{Po}$ $\left(T_{1 / 2}=3.10 \mathrm{~min}\right)-{ }^{214} \mathrm{~Pb}\left(T_{1 / 2}=26.8 \mathrm{~min}\right)$ chain, and 5.49 and $6.00 \mathrm{MeV} \alpha$-ray events could occur in the same time window in the V.H.E. energy range. This background is taken into account in the dummy data generation based on the measured ${ }^{222} \mathrm{Rn}$ activity of $\sim 8 \mathrm{mBq}$ in the active LXe volume [22]. The contributions from short-time consecutive decays in the ${ }^{238} \mathrm{U}$ and ${ }^{232} \mathrm{Th}$ chains contaminated in the detector material are turned out to be negligible. Figure 5 shows the distributions of the observed number of events in coincidence windows with various widths for the data of the pre-search window overlaid with the simulation. The simulated distributions well reproduce the data of the pre-search window.

Finally, the look-elsewhere effect due to the search using 4 energy windows is accounted for. For small $p$-values, this correction can be made by multiplying the $p$-value by the number of energy ranges, that is 4 . 

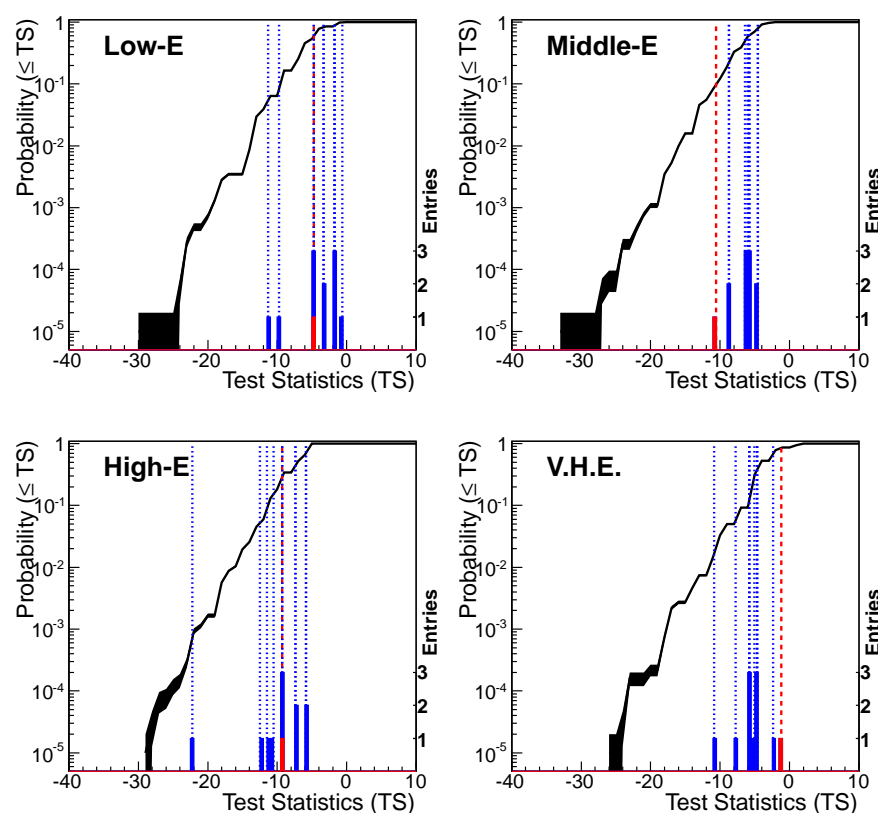

Figure 6: Distributions of the test statistics (TS) observed in each energy range for 10 binary black-hole mergers (blue dotted) and a binary neutron-star merger (red dashed). The black curve shows the probability of observing smaller TS value obtained from the 100,000 dummy data sets.

As the result of the model independent search, no coincidence time window with a global significance of more than $3 \sigma$ was found in the time range between -400 and $+10,000 \mathrm{~s}$ from the GW170817 event.

\subsection{Results for the binary black hole mergers}

The same analysis is performed for other $10 \mathrm{GW}$ events classified as the binary black hole mergers. Figure 6 shows the distributions of the TS observed in each energy range for 10 binary black-hole mergers and a binary neutron-star merger. Among them, a burst candidate with small probability of $P(\leq$ TS) $=7.8 \times 10^{-4}$ was found in the High-E energy range for the GW151012 event. As seen in Fig. 7 15 events are clustered within $2 \mathrm{~s}$ centered at $t-t_{\mathrm{GW}}=1802.95 \mathrm{~s}$. Figure 8 shows the test statistics as a function of the coincidence time width in the High-E energy range around $t-t_{\mathrm{GW} 151012}=1802.95 \mathrm{~s}$. The coincidence time width of $2 \mathrm{~s}$ gives the lowest TS value, and hence the most significant result. After considering the lookelsewhere effect of the 4 energy ranges, the global significance of this burst candidate identified in association with GW151012 is $3.0 \sigma$. Since we perform the analysis separately on the $11 \mathrm{GW}$ events, there is an additional look-elsewhere effect. The significance of finding such a burst candidate in any of the $11 \mathrm{GW}$ events is $2.1 \sigma$.

The energy and vertex position of those events are reconstructed based on a maximum-likelihood evaluation of the observed NPE of all the PMTs [19]. Note that the XMASS-I detector observes the LXe scintillation light, and therefore provides no directional information on detected particles. Figure 9 shows the reconstructed energy and radial position distributions

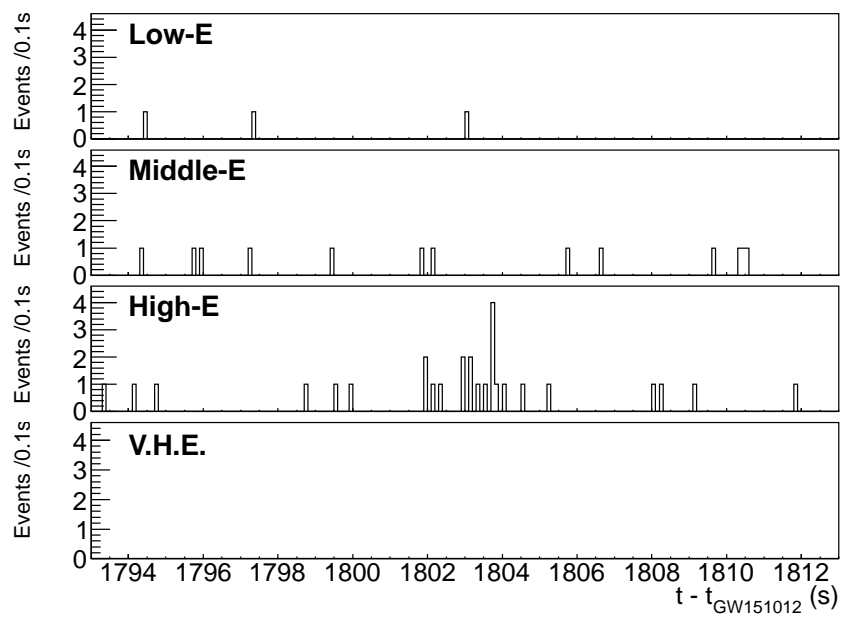

Figure 7: Event rate history within $\pm 10 \mathrm{~s}$ around the burst candidate found centered at $t-t_{\mathrm{GW}}=1802.95 \mathrm{~s}$ from GW151012 (October 12, 2015 09:54:43 UTC). From top to bottom, the Low-E, Middle-E, High-E, and V. H. E. samples are shown.

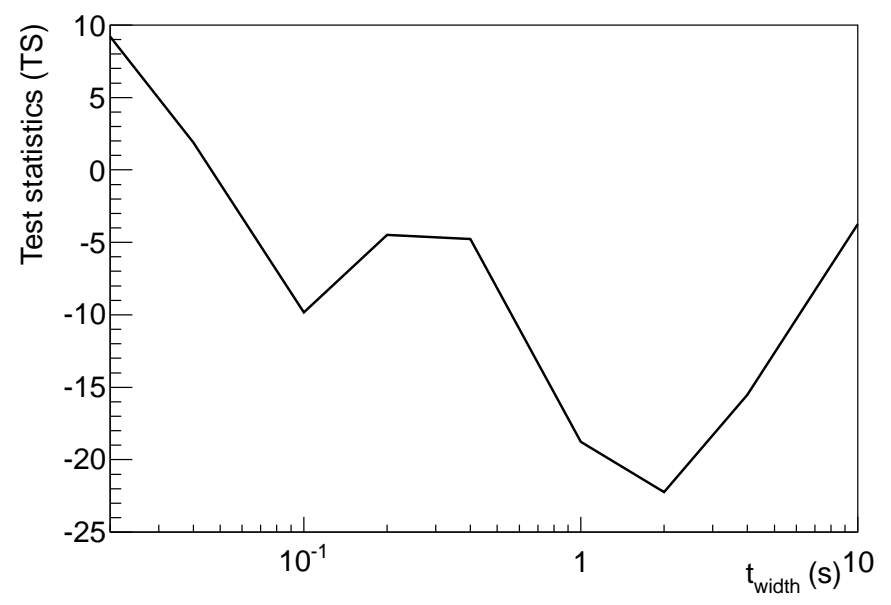

Figure 8: Test statistics (TS) as a function of coincidence time width $t_{\text {width }}$ in the High-E energy range around $t-t_{\mathrm{GW}}=1802.95 \mathrm{~s}$ from GW151012. Note that $t_{\text {width }}$ is scanned discretely at $0.02,0.04,0.1,0.2,0.4,1,2,4$, and $10 \mathrm{~s}$. 
of those 15 events overlaid with the ones for the background estimated using the pre-search window. The $p$-value of the Kolmogorov-Smirnov (KS) test for the energy and radial position distributions between those 15 events and the background are 0.87 and 0.96 , respectively. Therefore, no significant deviation from the background distributions is found.

To investigate whether the radial distribution of the burst candidate is consistent with that of potential signal uniformly distributed inside the detector, we overlay the radial distribution for uniformly distributed electron events (blue dashed in Fig. 9) using our GEANT4-based detector simulation. Since we do not have any signal model predicting the energy spectrum, a flat energy spectrum up to $3 \mathrm{MeV}$ is assumed. A dip at $(R / 42.5 \mathrm{~cm})^{3} \sim 0.7$ in the reconstructed radial distribution for the simulation is due to a slight position dependent bias in the radial direction. Note that the edge of the pentakisdodecahedral detector locates between $(R / 42.5 \mathrm{~cm})^{3}=0.83$ and 1. The $p$-value of the KS test for the uniformly distributed events is 0.055 .

We concluded that the radial distribution of the burst candidate is compatible with both that of the background and that of the uniform distribution since the obtained p-values of the KS test under both hypotheses are above 0.05 (or within $2 \sigma$ ). We also estimated the frequency that 15 events in the High-E energy region are observed within $2 \mathrm{~s}$ due to statistical fluctuation during the entire data-taking period of XMASS-I to be $0.21 \mathrm{yr}^{-1}$, based on the average event rate in the pre-search window.

\section{Constraints on neutrino fluence for GW170817}

For the GW170817 event, we also derive constraints on neutrino fluence for the sum of all neutrino flavors via CEvNS under the assumption of two types of neutrino energy distributions: a Fermi-Dirac spectrum with average neutrino energy of $20 \mathrm{MeV}$ and mono-energetic neutrinos in the range between 14 and $100 \mathrm{MeV}$. The relative difference in propagation time for GW and such neutrinos and GWs from the source, for example, at a $40 \mathrm{Mpc}$ distance is expected to be $<O(1) \mathrm{s}$. For this neutrino signal search, we use the $\pm 400 \mathrm{~s}$ search window, which is a similar size of the search window as the one used in searches by other neutrino detectors [5, 6, 7, 8, 9, 10, 11, 12].

The Fermi-Dirac energy distribution is expressed as

$$
f\left(E_{v}\right)=\frac{C}{\left(k_{\mathrm{B}} T\right)^{3}} \frac{E_{v}^{2}}{e^{E_{v} / k_{\mathrm{B}} T}+1},
$$

where

$$
C=\left(\int_{0}^{\infty} \frac{x^{2}}{e^{x}+1} \mathrm{~d} x\right)^{-1}=\frac{2}{3 \zeta(3)}
$$

is the normalization factor, $k_{\mathrm{B}}$ is the Boltzmann constant, $T$ is temperature, and the average energy of neutrinos is given by $\left\langle E_{v}\right\rangle \sim 3.15 T$.

The differential cross section of CEvNS is

$$
\frac{\mathrm{d} \sigma}{\mathrm{dE}_{\mathrm{nr}}}\left(E_{v}, E_{\mathrm{nr}}\right)=\frac{G_{F}^{2} M}{2 \pi} G_{V}^{2}\left[1+\left(1-\frac{E_{\mathrm{nr}}}{E_{v}}\right)^{2}-\frac{M E_{\mathrm{nr}}}{E_{v}^{2}}\right],
$$
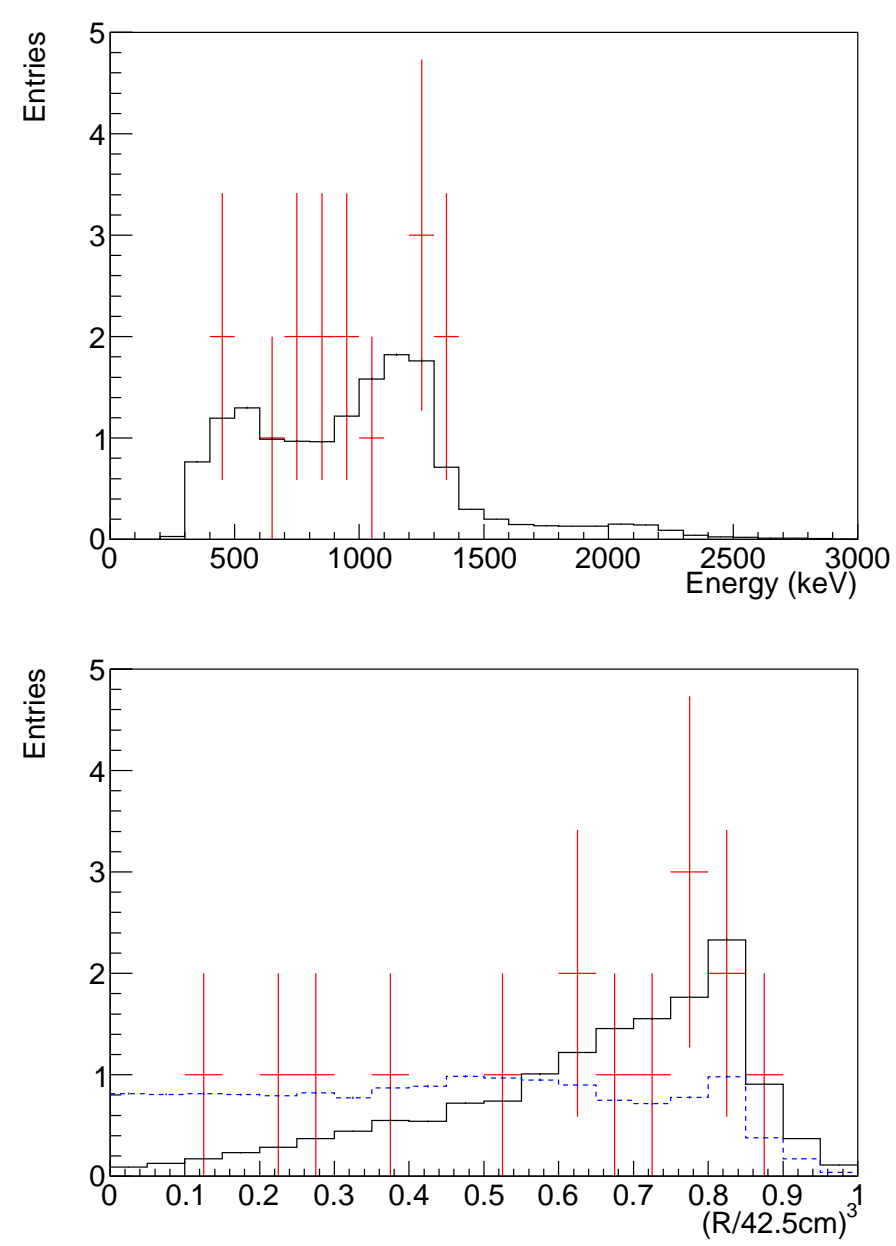

Figure 9: Reconstructed energy (top) and radial position (bottom) of the 15 events in the burst candidate overlaid with the distributions for background estimated using the pre-search window (black solid). The simulated radial distribution for the uniformly distributed events (blue dashed) is also shown for comparison. The distributions for background and the uniformly distributed events are normalized to the observed number of events. 


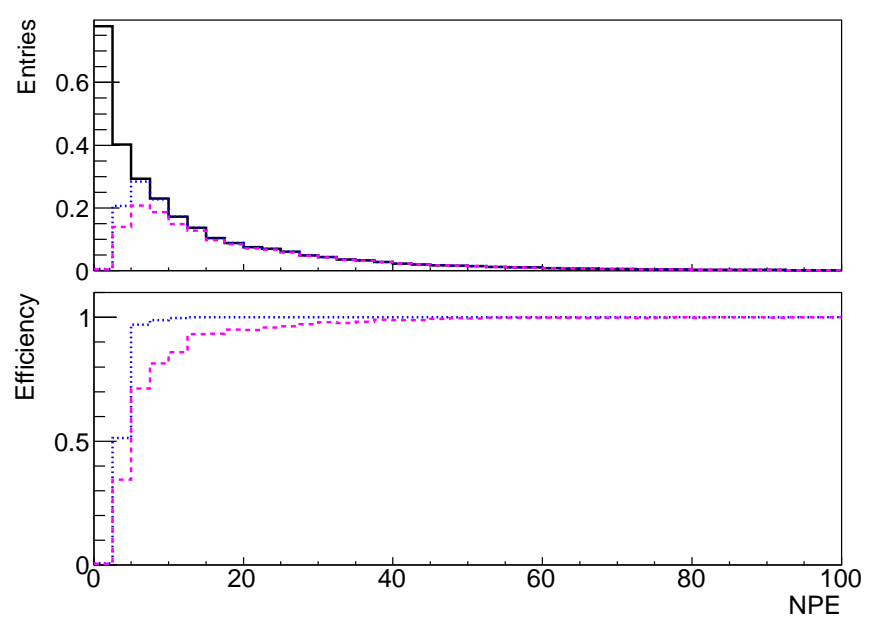

Figure 10: Simulated NPE spectra (top) and detection efficiency as a function of NPE (bottom) for the Fermi-Dirac spectrum with $\left\langle E_{\gamma}\right\rangle=20 \mathrm{MeV}$. The black solid, the blue dotted, and the magenta dashed histograms are without any cut, after the inner-detector trigger selection, and after the Cherenkov cut, respectively.

where $G_{F}$ is the Fermi constant, $M$ is the target nuclear mass, $E_{\mathrm{nr}}$ is the nuclear recoil energy, and

$$
G_{V}=\left[\left(\frac{1}{2}-2 \sin ^{2} \theta_{W}\right) Z-\frac{1}{2} N\right] F\left(q^{2}\right) .
$$

Here, $\theta_{\mathrm{W}}$ is the weak mixing angle, $Z$ and $N$ are the numbers of protons and neutrons in the nucleus, and $F\left(q^{2}\right)$ is the nuclear form factor, respectively. More detail for the calculation of the CEvNS interaction in XMASS-I can be found elsewhere [26].

Figure 10 shows the simulated NPE spectra and detection efficiency as a function of NPE for the Fermi-Dirac spectrum with $\left\langle E_{v}\right\rangle=20 \mathrm{MeV}$. The simulated neutrino events concentrate at low energy, and hence the Low-E sample (below $450 \mathrm{PE}$ ) is used to derive constraints on neutrino fluence. The detection efficiency crosses $50 \%$ at $4.5 \mathrm{PE}$ which corresponds to $3.8 \mathrm{keV}_{\mathrm{nr}}$.

No significant event burst is observed in the Low-E sample as described in the previous section, the $90 \%$ confidence level (CL) upper limit on neutrino fluence $\left(\Phi_{90}\right)$ is calculated by

$$
\Phi_{90}=\frac{N_{90}}{N_{T} \iint f\left(E_{v}\right) \frac{\mathrm{d} \sigma}{\mathrm{d} E_{\mathrm{nr}}}\left(E_{v}, E_{\mathrm{nr}}\right) \epsilon\left(E_{\mathrm{nr}}\right) \mathrm{dE}_{\nu} \mathrm{dE}_{\mathrm{nr}}}
$$

where $N_{T}$ is the number of target nuclei, $\epsilon\left(E_{\mathrm{nr}}\right)$ is the detection efficiency as a function of recoil energy and is estimated using our detector simulation. $N_{90}$ is the $90 \%$ CL upper limit on the number of signal events, derived from the relation

$$
\frac{\int_{0}^{N_{90}} P\left(\mu_{\text {sig }}+\mu_{\mathrm{bg}} \mid N_{\text {obs }}\right) \mathrm{d} \mu_{\text {sig }}}{\int_{0}^{\infty} P\left(\mu_{\text {sig }}+\mu_{\text {bg }} \mid N_{\text {obs }}\right) \mathrm{d} \mu_{\text {sig }}}=0.9
$$

where $P(\mu \mid N)$ is the Poisson probability, $N_{\text {obs }}$ is the observed number of events in the coincidence time window with a width $t_{\text {width }}$, and $\mu_{\text {sig }}$ and $\mu_{\text {bg }}$ are the average number of signal and background events, respectively. $\mu_{\mathrm{bg}}$ is estimated based on the

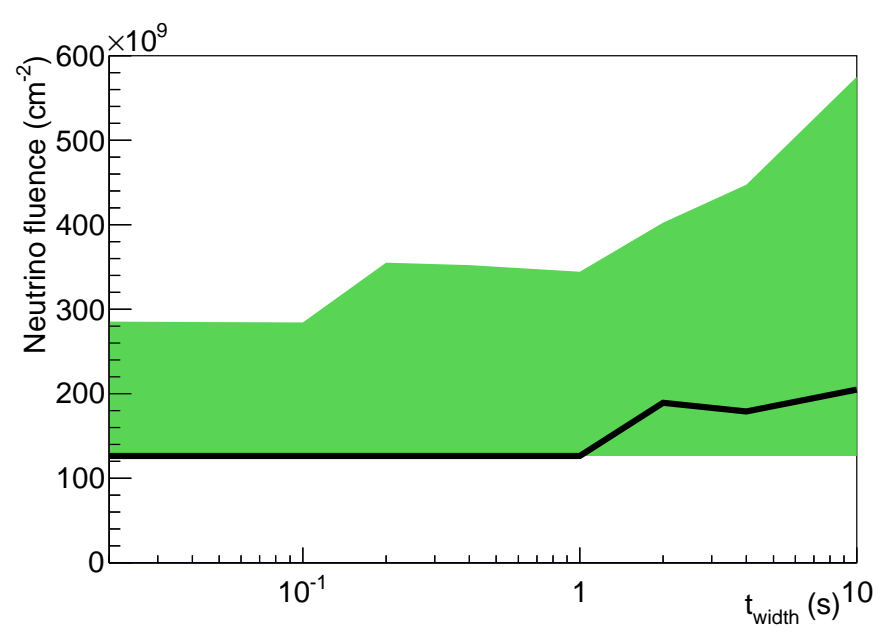

Figure 11: 90\% CL upper limits on neutrino fluence for GW170817 assuming the Fermi-Dirac spectrum with $\left\langle E_{v}\right\rangle=20 \mathrm{MeV}$ as a function of the coincidence time width $t_{\text {width. }}$ The black solid line shows the upper limit from the on-time window centered at $t=t_{\mathrm{GW}}$ with a width $t_{\mathrm{width}}$, and the green band represents the range of limits from the sliding window with a width $t_{\text {width }}$ within the $\pm 400 \mathrm{~s}$ search window. Note that $t_{\text {width }}$ is scanned discretely at $0.02,0.04,0.1,0.2,0.4$, $1,2,4$, and $10 \mathrm{~s}$.

average event rate estimated in the pre-search window, and we assume a uniform prior on $\mu_{\text {sig. }}$.

Figure 11 shows the $90 \%$ CL upper limits on neutrino fluence for the Fermi-Dirac spectrum with $\left\langle E_{v}\right\rangle=20 \mathrm{MeV}$ as a function of the coincidence time width $t_{\text {width }}$. The upper limit from the on-time window centered at $t=t_{\mathrm{GW}}$ with a width $t_{\mathrm{width}}$ is drawn as a line while the range of limits from the sliding window with a width $t_{\text {width }}$ within the $\pm 400 \mathrm{~s}$ search window is drawn as a band. The obtained upper limit from the on-time window was $(1.3-2.1) \times 10^{11} \mathrm{~cm}^{-2}$.

Figure 12 shows our $90 \% \mathrm{CL}$ upper limits on fluence for mono-energetic neutrinos as a function of neutrino energy between 14 and $100 \mathrm{MeV}$. Limits obtained by SuperKamiokande [11] are also shown. While their limits were derived utilizing the inverse beta decay $\left(\bar{v}_{e}+p \rightarrow e^{+}+n\right)$ for $\bar{v}_{e}$ and neutrino-electron elastic scattering $\left(v+e^{-} \rightarrow v+e^{-}\right)$for $v_{e}$ and $v_{\mu, \tau}$, our limits are for the sum of all the neutrino flavors utilizing CEvNS. The XMASS limit is comparable to the $v_{\mu, \tau}$ and $\bar{v}_{\mu, \tau}$ limits of Super-Kamiokande.

\section{Conclusion}

We conducted a search for event bursts in the XMASSI detector associated with $11 \mathrm{GW}$ events detected during LIGO/Virgo's $\mathrm{O} 1$ and $\mathrm{O} 2$ periods. We used the full $832 \mathrm{~kg}$ of xenon as an active target. Simple and loose cuts were applied to the data collected around the detection time of each GW event and the data were divided into four energy regions ranging from $\mathrm{keV}$ to $\mathrm{MeV}$. Without assuming any particular burst model, we looked for event bursts in sliding windows with various time width from 0.02 to $10 \mathrm{~s}$ and the search was conducted in a time window between -400 and $+10,000$ s from each $\mathrm{GW}$ 


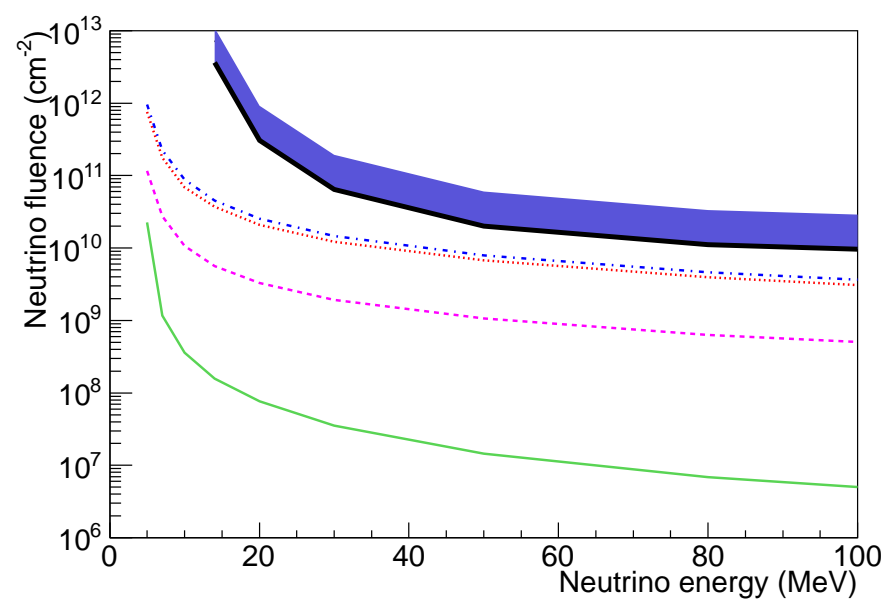

Figure 12: 90\% CL upper limits on mono-energetic neutrino fluence for GW170817 as a function of neutrino energy. The black solid line shows the upper limit from the on-time window, and the blue band represents the range of limits from the sliding window within the $\pm 400 \mathrm{~s}$ search window. Limits obtained by Super-Kamiokande [11] for $\bar{v}_{e}$ (green solid), $v_{e}$ (magenta dashed), $v_{\mu, \tau}$ (red dotted), and $\bar{v}_{\mu, \tau}$ (blue dash-dotted) are also shown.

event. For the binary neutron star merger GW170817, no significant event burst was observed in the XMASS-I detector, and hence we set $90 \%$ confidence level upper limits on neutrino fluence for the sum of all the neutrino flavors via coherent elastic neutrino-nucleus scattering. The obtained upper limit was $(1.3-2.1) \times 10^{11} \mathrm{~cm}^{-2}$ under the assumption of the Fermi-Dirac spectrum with the average neutrino energy of $20 \mathrm{MeV}$. The neutrino fluence limits for mono-energetic neutrinos in the energy range between 14 and $100 \mathrm{MeV}$ were also calculated. Among the other $10 \mathrm{GW}$ events detected as the binary black hole mergers, a burst candidate with a $3.0 \sigma$ significance was found at 1801.95-1803.95 s in the analysis for GW151012. However, the reconstructed energy and position distributions were consistent with those expected from the background. Considering the additional look-elsewhere effect of analyzing the $11 \mathrm{GW}$ events, the significance of finding such a burst candidate associated with any of them is $2.1 \sigma$.

\section{Acknowledgments}

We gratefully acknowledge the cooperation of the Kamioka Mining and Smelting Company. This work was supported by the Japanese Ministry of Education, Culture, Sports, Science and Technology, Grant-in-Aid for Scientific Research (19GS0204, 26104004, 26104007, and 26105505), the joint research program of the Institute for Cosmic Ray Research (ICRR), the University of Tokyo, and partially by the National Research Foundation of Korea Grant funded by the Korean Government (NRF-2011-220-C00006).

\section{References}

[1] B. P. Abbott et al., Phys. Rev. Lett. 116 (2016) 061102.
[2] B. P. Abbott et al., Phys. Rev. X 9 (2019) 031040.

[3] B. P. Abbott et al., Phys. Rev. Lett. 119 (2017) 161101.

[4] B. P. Abbott et al., Astrophys. J. 848 (2017) L12.

[5] S. Adrian-Martinez et al., Phys. Rev. D 93 (2016) 122010.

[6] A. Aab et al., Phys. Rev. D 94 (2016) 122007.

[7] A. Gando et al., Astrophys. J. 829 (2016) L34; A. Gando et al., Astrophys. J. 851 (2017) L22.

[8] K. Abe et al., Astrophys. J. 830 (2016) L11.

[9] M. Agostini et al., Astrophys. J. 850 (2017) 21.

[10] A. Albert et al., Astrophys. J. 850 (2017) L35.

[11] K. Abe et al., Astrophys. J. 857 (2018) L4.

[12] M. A. Acero et al., Phys. Rev. D 101 (2020), 112006.

[13] Y. Sekiguchi, K. Kiuchi, K. Kyutoku and M. Shibata, Phys. Rev. Lett. 107 (2011) 051102.

[14] K. Kyutoku and K. Kashiyama, Phys. Rev. D 97 (2018) 103001.

[15] S. S. Kimura, K. Murase, P. Meszaros and K. Kiuchi, Astrophys. J. 848 (2017) L4.

[16] K. Fang and B. D. Metzger, Astrophys. J. 849 (2017) 153.

[17] R. Perna, D. Lazzati and B. Giacomazzo, Astrophys. J. Lett. 821 (2016) L18.

[18] K. Kotera and J. Silk, Astrophys. J. Lett. 823 (2016) L29.

[19] K. Abe et al., Nucl. Instrum. Meth. A 716 (2013) 78.

[20] K. Abe et al., Phys. Rev. D 97 (2018) 102006.

[21] K. Abe et al., Phys. Lett. B 787 (2018) 153.

[22] K. Abe et al., Phys. Lett. B 789 (2019) 45.

[23] M. Kobayashi et al., Phys. Lett. B 795 (2019) 308.

[24] K. Abe et al., Phys. Lett. B 724 (2013) 46.

[25] N. Oka et al., Prog. Theor. Eep. Phys. 2017 (2017) 103 C01.

[26] K. Abe et al., Astropart. Phys. 89 (2017) 51.

[27] N. Y. Kim et al., Nucl. Instrum. Meth. A 784 (2015) 499 